\title{
A regularization algorithm for a splitting feasibility problem in Hilbert spaces
}

\author{
Abdul Latif ${ }^{\mathrm{a}}$, Xiaolong Qin ${ }^{\mathrm{b}, *}$ \\ ${ }^{a}$ Department of Mathematics, King Abdulaziz University, P. O. Box 80203, Jeddah-21589, Saudi Arabia. \\ ${ }^{b}$ Institute of Fundamental and Frontier Sciences, University of Electronic Science and Technology of China, Sichuan, China. \\ Communicated by Y. J. Cho
}

\begin{abstract}
In this article, we investigate a split feasibility problem via a regularization iterative algorithm. Strong convergence theorems of solutions for the split feasibility are established in the framework of Hilbert spaces. We also apply our main results to the split equality problem. (C)2017 All rights reserved.
\end{abstract}

Keywords: Metric projection, monotone operator, nonexpansive mapping, split feasibility problem, variational inequality. 2010 MSC: 47H05, 47H09, 90C33.

\section{Introduction}

In this paper, we always assume that $\mathrm{H}_{1}$ and $\mathrm{H}_{2}$ are real Hilbert spaces endowed with inner products and induced norms denoted by $\langle\cdot, \cdot\rangle$ and $\|\cdot\|$, respectively, while $H$ refers to as any of these spaces. Let $\mathrm{D}$ be a nonempty closed and convex subset of $\mathrm{H}$. Recall that $\operatorname{Proj}_{\mathrm{D}}^{\mathrm{H}}$ is said to be a metric projection from $\mathrm{H}$ onto D iff

$$
\left\|x-\operatorname{Proj}_{\mathrm{D}}^{\mathrm{H}} \mathrm{x}\right\| \leqslant\|x-y\|, \quad \forall x \in H, y \in \mathrm{D} .
$$

It is known that

$$
\left\langle x-y, \operatorname{Proj}_{D}^{\mathrm{H}} x-\operatorname{Proj}_{D}^{\mathrm{H}} y\right\rangle \geqslant\left\|\operatorname{Proj}_{D}^{\mathrm{H}} x-\operatorname{Proj}_{D}^{\mathrm{H}} y\right\|^{2}, \quad \forall x, y \in H .
$$

Moreover, $\operatorname{Proj}_{D}^{\mathrm{H}} \mathrm{x}$ is also characterized by the fact $\mathrm{P}_{\mathrm{D}} \mathrm{x} \in \mathrm{C}$ and

$$
\left\langle x-\operatorname{Proj}_{\mathrm{D}}^{\mathrm{H}} x, y-\operatorname{Proj}_{\mathrm{D}}^{\mathrm{H}} \mathrm{x}\right\rangle \leqslant 0,
$$

and

$$
\|x-y\|^{2} \geqslant\left\|x-\operatorname{Proj}_{D}^{\mathrm{H}} x\right\|^{2}+\left\|y-\operatorname{Proj}_{D}^{\mathrm{H}} \mathrm{x}\right\|^{2}, \quad \forall x \in H, y \in C .
$$

Recall that a mapping $M: H \rightarrow H$ is said to be contractive iff there exists a constant $k \in(0,1)$ such that

$$
\|M x-M y\| \leqslant k\|x-y\|, \quad \forall x, y \in H .
$$

\footnotetext{
*Corresponding author

Email addresses: alatif@kau.edu.sa (Abdul Latif), qxlxajh@163.com (Xiaolong Qin)
} 
Recall that $\mathrm{M}: \mathrm{H} \rightarrow \mathrm{H}$ is said to be contractive iff

$$
\|M x-M y\| \leqslant\|x-y\|, \quad \forall x, y \in H .
$$

Also, recall that $\mathrm{M}: \mathrm{H} \rightarrow \mathrm{H}$ is said to be averaged iff it can be written as the average of the identity mapping and a nonexpansive mapping, i.e., $M=(1-\alpha) I+\alpha \mathrm{N}$ where $\alpha \in(0,1)$ and $\mathrm{N}: \mathrm{H} \rightarrow \mathrm{H}$ is nonexpansive and $\mathrm{I}$ is the identity operator on $\mathrm{H}$. Recall that $\mathrm{M}: \mathrm{H} \rightarrow \mathrm{H}$ is said to be firmly nonexpansive iff

$$
\|M x-M y\|^{2} \leqslant\|x-y\|^{2}-\|x-y-(M x-M y)\|^{2}, \quad \forall x, y \in H .
$$

We note here that averaged mappings are nonexpansive. Further, firmly nonexpansive mappings are averaged. We also remark here that metric projections on nonempty closed and convex subsets are averaged. If $T=(1-\alpha) N+\alpha A$, where $A: H \rightarrow H$ is averaged, $N: H \rightarrow H$ is nonexpansive and $\alpha \in(0,1)$, then $T$ is averaged. The composite of finitely many averaged mappings is still averaged. Recently, fixed point methods of nonexpansive mappings have been studied for several convex optimization problems; see $[1,8,14,17]$ and the references therein.

Recall that a mapping $\mathrm{F}: \mathrm{H} \rightarrow \mathrm{H}$ is said to be monotone iff

$$
\langle F x-F y, x-y\rangle \geqslant 0, \quad \forall x, y \in H .
$$

Recall that $\mathrm{F}: \mathrm{H} \rightarrow \mathrm{H}$ is said to be inverse-strongly monotone iff there exists a constant $v>0$ such that

$$
\langle F x-F y, x-y\rangle \geqslant v\|F x-F y\|^{2}, \quad \forall x, y \in H .
$$

In such a case, we also say that $F$ is $v$-inverse-strongly monotone. Recall that $F: H \rightarrow H$ is said to be strongly monotone iff there exists a constant $v>0$ such that

$$
\langle F x-F y, x-y\rangle \geqslant v\|x-y\|^{2}, \quad \forall x, y \in H .
$$

In such a case, we also say that $F$ is $v$-strongly monotone. Recall that $F: H \rightarrow H$ is said to be Lipschitz continuous iff there exits $L>0$ such that

$$
\|F x-F y\| \leqslant L\|x-y\|, \quad \forall x, y \in H .
$$

In such case, we also say that $F$ is L-Lipschitz continuous. If $F$ is $v$-inverse-strongly monotone, then it is $\frac{1}{v}$-Lipschitz continuous and monotone. Let $\mathrm{F}$ be a (firmly) nonexpansive mapping and define a mapping $\mathrm{T}: \mathrm{H} \rightarrow \mathrm{H}$ by $\mathrm{T} x=(\mathrm{I}-\mathrm{F}) x$, for all $x \in \mathrm{H}$. Then $\mathrm{T}$ is $\frac{1}{2}$-inverse-strongly monotone. Recently, zero point problems of monotone (accretive) operators have been extensively investigated by many authors via fixed point methods; see $[2,3,10,15,16]$ and the references therein.

Let $\mathrm{C}$ and $\mathrm{Q}$ be nonempty closed convex subsets of $\mathrm{H}_{1}$ and $\mathrm{H}_{2}$, respectively. Let $\operatorname{Proj}_{\mathrm{C}} \mathrm{H}_{1}$ and $\operatorname{Proj}_{\mathrm{Q}} \mathrm{H}_{2}$ be the metric projections onto $\mathrm{C}$ and $\mathrm{Q}$, respectively. Recall that the split feasibility problem is to find a point $x \in \mathrm{H}_{1}$ such that

$$
x \in \mathrm{C}, \quad \mathrm{Ax} \in \mathrm{Q},
$$

where $A: H_{1} \rightarrow H_{2}$ is a bounded linear operator. From now on, we use Sol(SFP) to denote the solution set of the split feasibility problem, that is, $\operatorname{Sol}(\mathrm{SFP}):=\left\{x \in \mathrm{H}_{1}, x \in \mathrm{C}, \mathrm{A} x \in \mathrm{Q}\right\}$. The split feasibility problem is quite general. It includes many important problems, such as, variational inequality problems, complementary problems, equilibrium problems, as special cases.

In 1994, Censor and Elfving [6] first introduced the split feasibility problem in finite dimensional spaces. Since then, the split feasibility problem has been extensively studied by many authors due to its extensive applications in signal processing and image reconstruction; see [4] and the references therein. Recently, it is also found that the split feasibility problem could also be applied to study the intensitymodulated radiation therapy; see, for example, [6, 7] and the references therein. 
It is well-known that if Sol(SFP) is not empty, then the split feasibility problem is equivalent to a fixed point problem

$$
P_{C}\left(x-\gamma A^{*}\left(I-P_{Q}\right) A x\right)=x,
$$

where $\gamma>0$ is a constant and $A^{*}$ is the adjoint operator of $A$. The split feasibility problem has recently been investigated via fixed point methods; see $[9,11,13,18,20]$ and the references therein. Define a mapping $A^{\gamma}$ by

$$
A^{\gamma} x=x-\gamma A^{*}\left(I-P_{Q}\right) A x .
$$

Then (1.1) is reduced to $x=P_{C} A^{\gamma} \chi$. It is easy to see that $\operatorname{Fix}\left(A^{\gamma}\right)=A^{-1}(Q)$ and hence $\operatorname{Sol}(\operatorname{SFP})=$ $C \cap \operatorname{Fix}\left(A^{\gamma}\right)=\operatorname{Fix}\left(P_{C} A^{\gamma}\right)$ for sufficiently small $\gamma>0$; see Zhou and Wang [20] for the details.

The rest of the paper is organized as follows. Section 2 contains several useful lemmas. In Section 3 , we introduce a CQ type iterative for the split feasibility problem. A strong convergence theorem is established in the framework of Hilbert spaces. We also provide an application to the split equality problem.

\section{Preliminaries}

Lemma 2.1 ([19]). Let $\mathrm{H}$ be a Hilbert space. Then there exists a strictly increasing continuous convex function $\mathrm{W}:[0, \infty) \rightarrow[0, \infty)$ with $\mathrm{W}(0)=0$ such that

$$
a\|x\|^{2}+(1-a)\|y\|^{2} \geqslant\|a x+(1-a) y\|^{2}+(1-a) a W(\|x-y\|), \quad \forall a \in[0,1]
$$

for all $x, y \in B_{r}(0):=\{x \in H:\|x\| \leqslant r\}$, where $r$ is some positive real number. In particular, we have

$$
a\|x\|^{2}+(1-a)\|y\|^{2} \geqslant\|a x+(1-a) y\|^{2}, \quad \forall a \in[0,1] .
$$

Lemma 2.2 ([12]). Let $\left\{a_{n}\right\}$ be a sequence of nonnegative real numbers such that $a_{n+1} \leqslant\left(1-t_{n}\right) a_{n}+b_{n}+c_{n}$, for all $n \geqslant 0$, where $\left\{c_{n}\right\}$ is a sequence of nonnegative real numbers, $\left\{t_{n}\right\} \subset(0,1)$, and $\left\{b_{n}\right\}$ is a sequence of real numbers. Assume that

(a) $\limsup _{n \rightarrow \infty} \frac{b_{n}}{t_{n}} \leqslant 0, \sum_{n=0}^{\infty} t_{n}=\infty$;

(b) $\sum_{n=0}^{\infty} c_{n}<\infty$.

Then $\lim _{n \rightarrow \infty} a_{n}=0$.

The following two lemmas are not hard to derive.

Lemma 2.3. Let $\operatorname{Proj}_{\mathrm{C}}^{\mathrm{H}}: \mathrm{H} \rightarrow \mathrm{C}$ be the metric projection from $\mathrm{H}$ on a nonempty, closed, and convex subset of $\mathrm{C}$. Then the following conclusions hold true

(a) $\left\langle\mathrm{P}_{\mathrm{C}}^{\mathrm{H}} \mathrm{x}-\mathrm{P}_{\mathrm{C}}^{\mathrm{H}} \mathrm{y}, \mathrm{x}-\mathrm{y}\right\rangle \geqslant\left\|\mathrm{P}_{\mathrm{C}}^{\mathrm{H}} \mathrm{x}-\mathrm{P}_{\mathrm{C}}^{\mathrm{H}} \mathrm{y}\right\|^{2}, x, y \in \mathrm{H}$.

(b) Given $\mathrm{x} \in \mathrm{H}$ and $z \in \mathrm{C}$, then $z=\mathrm{P}_{\mathrm{C}}^{\mathrm{H}} \mathrm{x}$ iff there holds the inequality $\langle x-z, y-z\rangle \leqslant 0, \mathrm{y} \in \mathrm{C}$.

(c) $\left\|\mathrm{P}_{\mathrm{C}}^{\mathrm{H}} \mathrm{x}-\mathrm{P}_{\mathrm{C}}^{\mathrm{H}} \mathrm{y}\right\|^{2} \leqslant\|x-y\|^{2}-\left\|\left(\mathrm{I}-\mathrm{P}_{\mathrm{C}}^{\mathrm{H}}\right) \mathrm{x}-\left(\mathrm{I}-\mathrm{P}_{\mathrm{C}}^{\mathrm{H}}\right) \mathrm{y}\right\|^{2}, \forall x, y \in \mathrm{H}$.

(d) $\left\langle\left(\mathrm{I}-\mathrm{P}_{\mathrm{C}}^{\mathrm{H}}\right) \mathrm{x}-\left(\mathrm{I}-\mathrm{P}_{\mathrm{C}}^{\mathrm{H}}\right) \mathrm{y}, \mathrm{x}-\mathrm{y}\right\rangle \geqslant\left\|\left(\mathrm{I}-\mathrm{P}_{\mathrm{C}}^{\mathrm{H}}\right) \mathrm{x}-\left(\mathrm{I}-\mathrm{P}_{\mathrm{C}}^{\mathrm{H}}\right) y\right\|^{2}, \forall x, y \in \mathrm{H}$.

Lemma 2.4. Let $\mathrm{H}$ be a Hilbert space. Then the following inequality holds.

$$
\|x+y\|^{2}=\|x\|^{2}+\|y\|^{2}+2\langle x, y\rangle \leqslant\|x\|^{2}+2\langle y, x+y\rangle .
$$

\section{Main results}

Theorem 3.1. Let $\mathrm{C}$ and $\mathrm{Q}$ be two nonempty, closed, and convex subsets of real Hilbert spaces $\mathrm{H}_{1}$ and $\mathrm{H}_{2}$, respectively. Let Proj $\mathrm{H}_{\mathrm{C}}^{\mathrm{H}_{1}}$ and Proj $_{\mathrm{O}}^{\mathrm{H}_{2}}$ be the metric projections onto $\mathrm{C}$ and $Q$, respectively. Let $\mathrm{A}: \mathrm{H}_{1} \rightarrow \mathrm{H}_{2}$ be a bounded linear operator and let $\mathrm{f}: \mathrm{H}_{1} \rightarrow \mathrm{H}_{1}$ be a $\mathrm{k}$-contractive mapping. Assume that $\operatorname{Sol}(\mathrm{SFP}) \neq \emptyset$. Let $\left\{\mathrm{x}_{\mathrm{n}}\right\}$ be a sequence generated in the following iterative algorithm

$$
x_{1} \in H, \quad x_{n+1}=\operatorname{Proj}_{C}^{\mathrm{H}_{1}}\left(\alpha_{n} f\left(x_{n}\right)+\left(1-\alpha_{n}\right)\left(x_{n}-\beta_{n} A^{*}\left(I-\operatorname{Proj}_{Q}^{\mathrm{H}_{2}}\right) A x_{n}\right)\right),
$$

where $\left\{\alpha_{n}\right\}$ is a sequence in $(0,1)$ such that $\lim _{n \rightarrow \infty} \alpha_{n}=0, \sum_{n=1}^{\infty} \alpha_{n}=\infty$ and $\sum_{n=1}^{\infty}\left|\alpha_{n}-\alpha_{n+1}\right|<\infty$ and 
$\left\{\beta_{n}\right\}$ is a sequence such that $\sum_{n=1}^{\infty}\left|\beta_{n}-\beta_{n+1}\right|<\infty, 0<p \leqslant \beta_{n} \leqslant q<\frac{2}{\|A\|^{2}}$, where $p$ and $q$ are two real numbers. Then $\left\{x_{n}\right\}$ converges strongly to a point $x^{*}$ in $\operatorname{Sol}(\mathrm{SFP})$ and $x^{*}=\mathrm{P}_{\mathrm{Sol}(\mathrm{SFP})} \mathrm{f}\left(\mathrm{x}^{*}\right)$.

Proof. Since Sol(SFP) is nonempty, closed, and convex, we see that projection $\mathrm{P}_{\mathrm{Sol}(\mathrm{SFP})} \mathrm{x}, \forall x \in \mathrm{H}_{1}$ onto $\mathrm{Sol}(\mathrm{SFP})$ is well-defined. Since Proj $\mathrm{Sol}_{\mathrm{S}(\mathrm{SFP})} \mathrm{f}$ is $\mathrm{K}$-contractive, we see that $\mathrm{P}_{\mathrm{Sol}(\mathrm{SFP})} f$ has a unique fixed point. Next, we use $x^{*}$ to denote the unique fixed point, that is, $x^{*}=\operatorname{Proj}_{\text {Sol }(S F P)}^{\mathrm{H}_{1}} f\left(x^{*}\right)$.

Define a mapping $\mathrm{F}: \mathrm{H}_{1} \rightarrow \mathrm{H}_{1}$ by $\mathrm{Fx}=\mathrm{A}^{*}\left(\mathrm{I}-\operatorname{Proj}_{\mathrm{Q}}^{\mathrm{H}_{2}}\right) A x$. Then (3.1) becomes

$$
x_{1} \in H, \quad x_{n+1}=\operatorname{Proj}_{C}^{H_{1}}\left(\alpha_{n} f\left(x_{n}\right)+\left(1-\alpha_{n}\right)\left(x_{n}-\beta_{n} A F x_{n}\right)\right) .
$$

Using Lemma 2.3, we have

$$
\begin{aligned}
\langle F x-F y, x-y\rangle & =\left\langle\left(I-\operatorname{Proj}_{Q}^{\mathrm{H}_{2}}\right) A x-\left(I-\operatorname{Proj}_{\mathrm{Q}}^{\mathrm{H}_{2}}\right) A y, A x-A y\right\rangle \\
& \geqslant\left\|\left(I-\operatorname{Proj}_{\mathrm{Q}}^{\mathrm{H}_{2}}\right) A x-\left(I-\operatorname{Proj}_{\mathrm{Q}}^{\mathrm{H}_{2}}\right) A y\right\|^{2} \\
& \geqslant \frac{1}{\|A\|^{2}}\left\|A^{*}\left(I-\operatorname{Proj}_{\mathrm{Q}}^{\mathrm{H}_{2}}\right) A x-A^{*}\left(I-\operatorname{Proj}_{\mathrm{Q}}^{\mathrm{H}_{2}}\right) A y\right\|^{2} \\
& =\frac{1}{\|A\|^{2}}\|F x-F y\|^{2} .
\end{aligned}
$$

This shows that $F$ is $\frac{1}{\|A\|^{2}}$-inverse-strongly monotone. Next, we prove $F^{-1}(0)=A^{-1}(Q)$. For all $x \in$ $A^{-1}(Q)$, we find from the definition of $F$ that $A^{-1}(Q) \subset F^{-1}(0)$. Next, we prove $F^{-1}(0)=A^{-1}(Q)$. It is easy to see that $A^{-1}(Q) \subset F^{-1}(0)$. Letting $x \in F^{-1}(0)$, we have $F x=0$. Since the split feasibility problem is consistent, we can take a point $y \in \operatorname{Sol}(S F P)$. This implies $A y=\operatorname{Proj}_{\mathrm{Q}}^{\mathrm{H}_{2}} A y$. Hence, $F y=0$. Using (3.2), we have

$$
\left\|\left(\mathrm{I}-\operatorname{Proj}_{\mathrm{Q}}^{\mathrm{H}_{2}}\right) A x\right\|^{2} \leqslant\langle\mathrm{Fx}-\mathrm{Fy}, x-\mathrm{y}\rangle=0,
$$

which implies that $x \in A^{-1}(Q)$, that is, $F^{-1}(0) \subset A^{-1}(Q)$. This shows that $F^{-1}(0)=A^{-1}(Q)$. Putting $y_{n}=x_{n}-\beta_{n} F x_{n}$, we have

$$
\begin{aligned}
\left\|y_{n}-x^{*}\right\|^{2} & =\left\|\beta_{n}\left(F x_{n}-F x^{*}\right)-\left(x_{n}-x^{*}\right)\right\|^{2} \\
& =\beta_{n}^{2}\left\|F x_{n}-F x^{*}\right\|^{2}-2 \beta_{n}\left\langle F x_{n}-F x^{*}, x_{n}-x^{*}\right\rangle+\left\|x_{n}-x^{*}\right\|^{2} \\
& \leqslant \beta_{n}^{2}\left\|F x_{n}\right\|^{2}-\frac{2 \beta_{n}}{\|A\|^{2}}\left\|F x_{n}\right\|^{2}+\left\|x_{n}-x^{*}\right\|^{2} \\
& =\left\|x_{n}-x^{*}\right\|^{2}-\left(\frac{2 \beta_{n}}{\|A\|^{2}}-\beta_{n}^{2}\right)\left\|F x_{n}\right\|^{2} .
\end{aligned}
$$

Since $0<p \leqslant \beta_{n} \leqslant q<\frac{2}{\|A\|^{2}}$, we have $\left\|y_{n}-x^{*}\right\| \leqslant\left\|x_{n}-x^{*}\right\|$. This implies that

$$
\begin{aligned}
\left\|x_{n+1}-x^{*}\right\| & =\left\|P_{C}\left(\alpha_{n} f\left(x_{n}\right)+\left(1-\alpha_{n}\right) y_{n}\right)-P_{C} x^{*}\right\| \\
& \leqslant\left\|\alpha_{n}\left(f\left(x_{n}\right)-x^{*}\right)+\left(1-\alpha_{n}\right)\left(y_{n}-x^{*}\right)\right\| \\
& \leqslant \alpha_{n}\left\|f\left(x_{n}\right)-f\left(x^{*}\right)\right\|+\alpha_{n}\left\|f\left(x^{*}\right)-x^{*}\right\|+\left(1-\alpha_{n}\right)\left\|y_{n}-x^{*}\right\| \\
& \leqslant \alpha_{n}\left\|f\left(x^{*}\right)-x^{*}\right\|+\left(1-\alpha_{n}(1-\kappa)\right)\left\|x_{n}-x^{*}\right\| \\
& \leqslant \max \left\{\frac{\left\|f\left(x^{*}\right)-x^{*}\right\|}{1-\kappa},\left\|x_{n}-x^{*}\right\|\right\} .
\end{aligned}
$$

By mathematical induction, we find that

$$
\left\|x_{n+1}-x^{*}\right\| \leqslant \max \left\{\frac{\left\|f\left(x^{*}\right)-x^{*}\right\|}{1-\kappa},\left\|x_{1}-x^{*}\right\|\right\} .
$$


This shows that $\left\{x_{n}\right\}$ is bounded. Since $F$ is $\frac{1}{\|\mathcal{A}\|^{2}}$-inverse-strongly monotone, we have

$$
\begin{aligned}
\left\|\left(I-\beta_{n} F\right) x-\left(I-\beta_{n} F\right) y\right\|^{2} & =\left\|\beta_{n}(F x-F y)-(x-y)\right\|^{2} \\
& =\|x-y\|^{2}-2 \beta_{n}\langle F x-F y, x-y\rangle+\beta_{n}^{2}\|F x-F y\|^{2} \\
& \leqslant\|x-y\|^{2}-\frac{2 \beta_{n}}{\|A\|^{2}}\|F x-F y\|^{2}+\beta_{n}^{2}\|F x-F y\|^{2} \\
& =\|x-y\|^{2}-\left(\frac{2 \beta_{n}}{\|A\|^{2}}-\beta_{n}^{2}\right)\|F x-F y\|^{2} .
\end{aligned}
$$

Since $0<p \leqslant \beta_{n} \leqslant q<\frac{2}{\|A\|^{2}}$, we find that $\left(I-\beta_{n} F\right)$ is nonexpansive. It follows that

$$
\begin{aligned}
\left\|x_{n+1}-x_{n}\right\| \leqslant & \left\|\left(\alpha_{n} f\left(x_{n}\right)+\left(1-\alpha_{n}\right) y_{n}\right)-\left(\alpha_{n-1} f\left(x_{n-1}\right)+\left(1-\alpha_{n-1}\right) y_{n-1}\right)\right\| \\
\leqslant & \alpha_{n}\left\|f\left(x_{n}\right)-f\left(x_{n-1}\right)\right\|+\left(1-\alpha_{n}\right)\left\|y_{n}-y_{n-1}\right\|+\left|\alpha_{n}-\alpha_{n-1}\right|\left\|f\left(x_{n-1}\right)-y_{n-1}\right\| \\
\leqslant & \alpha_{n} k\left\|x_{n}-x_{n-1}\right\|+\left(1-\alpha_{n}\right)\left\|\left(I-\beta_{n} F\right) x_{n}-\left(I-\beta_{n} F\right) x_{n-1}\right\| \\
& +\left(1-\alpha_{n}\right)\left\|\left(I-\beta_{n} F\right) x_{n-1}-\left(I-\beta_{n-1} F\right) x_{n-1}\right\|+\left|\alpha_{n}-\alpha_{n-1}\right|\left\|f\left(x_{n-1}\right)-y_{n-1}\right\| \\
\leqslant & \left(1-(1-\kappa) \alpha_{n}\right)\left\|x_{n}-x_{n-1}\right\|+\left|\beta_{n}-\beta_{n-1}\right|\left\|F x_{n-1}\right\|+\left|\alpha_{n}-\alpha_{n-1}\right|\left\|f\left(x_{n-1}\right)-y_{n-1}\right\| .
\end{aligned}
$$

Using Lemma 2.2, we find that

$$
\lim _{n \rightarrow \infty}\left\|x_{n+1}-x_{n}\right\|=0
$$

Since $\|\cdot\|^{2}$ is convex, we find from (3.3) that

$$
\begin{aligned}
\left\|x_{n+1}-x^{*}\right\|^{2} & \leqslant\left\|\left(\alpha_{n} f\left(x_{n}\right)+\left(1-\alpha_{n}\right) y_{n}\right)-x^{*}\right\|^{2} \\
& \leqslant \alpha_{n}\left\|f\left(x_{n}\right)-x^{*}\right\|^{2}+\left(1-\alpha_{n}\right)\left\|y_{n}-x^{*}\right\|^{2} \\
& \leqslant \alpha_{n}\left\|f\left(x_{n}\right)-x^{*}\right\|^{2}+\left(1-\alpha_{n}\right)\left\|x_{n}-x^{*}\right\|^{2}-\left(1-\alpha_{n}\right)\left(\frac{2 \beta_{n}}{\|A\|^{2}}-\beta_{n}^{2}\right)\left\|F x_{n}\right\|^{2} .
\end{aligned}
$$

It follows that

$$
\begin{aligned}
\left(1-\alpha_{n}\right) \beta_{n}\left(\frac{2}{\|A\|^{2}}-\beta_{n}\right)\left\|F x_{n}\right\|^{2} & \leqslant \alpha_{n}\left\|f\left(x_{n}\right)-x^{*}\right\|^{2}+\left(1-\alpha_{n}\right)\left\|x_{n}-p\right\|^{2}-\left\|x_{n+1}-p\right\|^{2} \\
& \leqslant \alpha_{n}\left\|f\left(x_{n}\right)-x^{*}\right\|^{2}+\left\|x_{n}-p\right\|^{2}-\left\|x_{n+1}-p\right\|^{2} \\
& \leqslant \alpha_{n}\left\|f\left(x_{n}\right)-x^{*}\right\|^{2}+\left(\left\|x_{n}-p\right\|+\left\|x_{n+1}-p\right\|\right)\left\|x_{n+1}-x_{n}\right\| .
\end{aligned}
$$

Using the restrictions imposed on $\left\{\alpha_{n}\right\}$ and $\left\{\beta_{n}\right\}$, we find from (3.4) that $\lim _{n \rightarrow \infty}\left\|F x_{n}\right\|=0$. This implies $\lim _{n \rightarrow \infty}\left\|x_{n}-y_{n}\right\|=0$. Since $F$ is Lipschitz continuous, we find that $\lim _{n \rightarrow \infty}\left\|F y_{n}\right\|=0$.

We are now in a position to show that

$$
\limsup _{n \rightarrow \infty}\left\langle f\left(x^{*}\right)-x^{*}, y_{n}-x^{*}\right\rangle \leqslant 0 .
$$

Take a subsequence $\left\{y_{n_{j}}\right\}$ of $\left\{y_{n}\right\}$ such that

$$
\limsup _{n \rightarrow \infty}\left\langle f\left(x^{*}\right)-x^{*}, y_{n}-x^{*}\right\rangle=\lim _{j \rightarrow \infty}\left\langle f\left(x^{*}\right)-x^{*}, y_{n_{j}}-x^{*}\right\rangle .
$$

Without loss of generality, we may assume that $\left\{y_{n_{j}}\right\}$ converges weakly to $\widehat{x}$. We find that $\widehat{x} \in F^{-1}(0)$. Indeed, Since $F$ is $\frac{1}{\|A\|^{2}}$-inverse-strongly monotone, we have

$$
\|A\|^{2}\left\langle F y_{n_{j}}-F \hat{x}, x_{n_{j}}-\widehat{x}\right\rangle \geqslant\left\|F y_{n_{j}}-F \hat{x}\right\|^{2} .
$$

Letting $j \rightarrow \infty$ in (3.5), we arrive at

$$
0 \geqslant\|\mathrm{~F} \widehat{x}\|^{2},
$$

which means that $F \hat{x}=0$. This further implies that

$$
\limsup _{n \rightarrow \infty}\left\langle f\left(x^{*}\right)-x^{*}, y_{n}-x^{*}\right\rangle=\lim _{j \rightarrow \infty}\left\langle f\left(x^{*}\right)-x^{*}, y_{n_{j}}-x^{*}\right\rangle \leqslant 0 .
$$


Finally, we prove $x_{n} \rightarrow x^{*}$. Using Lemma 2.4, we find that

$$
\begin{aligned}
\left\|x_{n+1}-x^{*}\right\|^{2} \leqslant & \left\|\left(1-\alpha_{n}\right)\left(y_{n}-x^{*}\right)+\alpha_{n}\left(f\left(x_{n}\right)-x^{*}\right)\right\|^{2} \\
= & \left(1-\alpha_{n}\right)^{2}\left\|y_{n}-x^{*}\right\|^{2}+\alpha_{n}^{2}\left\|f\left(x_{n}\right)-x^{*}\right\|^{2}+2 \alpha_{n}\left(1-\alpha_{n}\right)\left\langle y_{n}-x^{*}, f\left(x_{n}\right)-x^{*}\right\rangle \\
\leqslant & \left(1-2 \alpha_{n}+\alpha^{2}\right)\left\|y_{n}-x^{*}\right\|^{2}+\alpha_{n}^{2}\left\|f\left(x_{n}\right)-x^{*}\right\|^{2}+2 \alpha_{n}\left(1-\alpha_{n}\right)\left\|y_{n}-x^{*}\right\|\left\|f\left(x_{n}\right)-f\left(x^{*}\right)\right\| \\
& +2 \alpha_{n}\left(1-\alpha_{n}\right)\left\langle y_{n}-x^{*}, f\left(x^{*}\right)-x^{*}\right\rangle \\
\leqslant & \left(1-2 \alpha_{n}+\alpha^{2}+2 \alpha_{n}\left(1-\alpha_{n}\right) \kappa\right)\left\|x_{n}-x^{*}\right\|^{2}+\alpha_{n}^{2}\left\|f\left(x_{n}\right)-x^{*}\right\|^{2} \\
& +2 \alpha_{n}\left(1-\alpha_{n}\right)\left\langle y_{n}-x^{*}, f\left(x^{*}\right)-x^{*}\right\rangle \\
= & \left(1-\alpha_{n}\left(2-\alpha-2\left(1-\alpha_{n}\right) \kappa\right)\right)\left\|x_{n}-x^{*}\right\|^{2}+\alpha_{n}\left(\alpha_{n}\left\|f\left(x_{n}\right)-x^{*}\right\|^{2}\right. \\
& \left.+2\left(1-\alpha_{n}\right)\left\langle y_{n}-x^{*}, f\left(x^{*}\right)-x^{*}\right\rangle\right) \\
= & \left(1-\alpha_{n}\left(2-\alpha-2\left(1-\alpha_{n}\right) \kappa\right)\right)\left\|x_{n}-x^{*}\right\|^{2} \\
& +\alpha_{n}\left(2-\alpha-2\left(1-\alpha_{n}\right) \kappa\right) \frac{\alpha_{n}\left\|f\left(x_{n}\right)-x^{*}\right\|^{2}+2\left(1-\alpha_{n}\right)\left\langle y_{n}-x^{*}, f\left(x^{*}\right)-x^{*}\right\rangle}{2-\alpha-2\left(1-\alpha_{n}\right) \kappa} .
\end{aligned}
$$

Using Lemma 2.2, we find that $x_{n} \rightarrow x^{*}$ as $n \rightarrow \infty$. This completes the proof.

Let $\mathrm{H}_{1}, \mathrm{H}_{2}$, and $\mathrm{H}_{3}$ be real Hilbert spaces, let $\mathrm{C} \subset \mathrm{H}_{1}$ and $\mathrm{Q} \subset \mathrm{H}_{2}$ be two nonempty, closed, and convex sets, and let $A: \mathrm{H}_{1} \rightarrow \mathrm{H}_{3}$ and $\mathrm{B}: \mathrm{H}_{2} \rightarrow \mathrm{H}_{3}$ be two bounded linear operators. Recall that the split equality problem is to

$$
\text { find } x \in C \text { and } y \in Q \text { such that } A x=B y \text {. }
$$

We use $\Omega$ to denote the solution set of the split equality problem, which was first introduced and studied by Moudafi and Al-Shemas [13]. By virtue of the product space techniques, we can convert the split equality problem to another specific split feasibility problem. To see this, set $S=C \times Q$ and define

$$
\mathrm{G}=[\mathrm{A},-\mathrm{B}], \quad \omega=[x, y]^{\top} .
$$

With these notations, we know that solving the split equality problem is equivalent to finding a point $\omega \in S$ such that $G \omega=0$. Assuming that the split equality problem is consistent, i.e., $\Omega \neq \emptyset$, then it is not hard to see that $\omega \in S$ solves the split equality problem if and only if it solves the operator equation $\mathrm{G}^{*} \mathrm{Gw}=0$, where $\mathrm{G}^{*}$ is the adjoint operator of $\mathrm{G}$. It is clear that $\mathrm{G}^{*} \mathrm{G}: \mathrm{H}_{1} \times \mathrm{H}_{2} \rightarrow \mathrm{H}_{1} \times \mathrm{H}_{2}$ is $\frac{1}{\|\mathrm{G}\|^{2}}$-inverse strongly monotone. By using Theorem 3.1, we deduce immediately the following result.

Corollary 3.2. Let $\mathrm{H}_{1}, \mathrm{H}_{2}$, and $\mathrm{H}_{3}$ be real Hilbert space. Let $\mathrm{C} \subset \mathrm{H}_{1}$ and $\mathrm{Q} \subset \mathrm{H}_{2}$ be two nonempty, closed, and convex sets of real Hilbert spaces $\mathrm{H}_{1}$ and $\mathrm{H}_{2}$, respectively. Let $\mathrm{A}: \mathrm{H}_{1} \rightarrow \mathrm{H}_{3}$ and $\mathrm{B}: \mathrm{H}_{2} \rightarrow \mathrm{H}_{3}$ be two bounded linear operators. Let $\mathrm{f}: \mathrm{H}_{1} \rightarrow \mathrm{H}_{1}$ be a $\mathrm{k}$-contractive mapping. Suppose that the $\Omega \neq \emptyset$ is consistent. Let $\left\{\alpha_{n}\right\}$ and $\left\{\beta_{n}\right\}$ be given as in Theorem 3.1. Let a sequence $\left\{\omega_{n}\right\}$ be generated by the algorithm

$$
\omega_{1} \in H_{1} \times H_{2}, \quad \omega_{n+1}=\operatorname{Proj}_{S}\left[\alpha_{n} f\left(\omega_{n}\right)+\left(1-\alpha_{n}\right)\left(\omega_{n}-\beta_{n} G^{*} G \omega_{n}\right)\right], \quad n \geqslant 1 .
$$

Then $\left\{\omega_{n}\right\}$ converges in norm to $\omega^{*}=\mathrm{P}_{\Omega} \mathrm{f}\left(\omega^{*}\right)$, that is, $\omega^{*}$ is a unique solution of the variational inequality

$$
\left\langle f\left(\omega^{*}\right)-\omega^{*}, \omega^{*}-y\right\rangle \geqslant 0, \quad \forall y \in \Omega \text {. }
$$

\section{Acknowledgment}

This work was supported by the Deanship of Scientific Research (DSR), King Abdulaziz University, Jeddah, under grant D-72-130-1438. The authors, therefore, gratefully acknowledge the DSR technical and financial support. 


\section{References}

[1] I. K. Argyros, S. George, S. M. Erappa, Expanding the applicability of the generalized Newton method for generalized equations, Commun. Optim. Theory, 2017 (2017), 12 pages. 1

[2] B. A. Bin Dehaish, A. Latif, H. O Bakodah, X.-L. Qin, A regularization projection algorithm for various problems with nonlinear mappings in Hilbert spaces, J. Inequal. Appl., 2015 (2015), 14 pages. 1

[3] B. A. Bin Dehaish, X.-L. Qin, A. Latif, H. Bakodah, Weak and strong convergence of algorithms for the sum of two accretive operators with applications, J. Nonlinear Convex Anal., 16 (2015), 1321-1336. 1

[4] C. Byrne, A unified treatment of some iterative algorithms in signal processing and image reconstruction, Inverse Problems, 20 (2004), 103-120. 1

[5] Y. Censor, T. Bortfeld, B. Martin, A. Trofimov, A unified approach for inversion problems in intensity-modulated radiation therapy, Phys. Med. Biol., 51 (2006), 2353-2365.

[6] Y. Censor, T. Elfving, A multiprojection algorithm using Bregman projections in a product space, Numer. Algorithms, 8 (1994), 221-239. 1

[7] Y. Censor, T. Elfving, N. Kopf, T. Bortfeld, The multiple-sets split feasibility problem and its applications for inverse problems, Inverse Problems, 21 (2005), 2071-2084. 1

[8] J. W. Chen, E. Kobis, M. A. Kobis, J.-C. Yao, Optimality conditions for solutions of constrained inverse vector variational inequalities by means of nonlinear scalarization, J. Nonlinear Var. Anal., 1 (2017), 145-158. 1

[9] S. Y. Cho, B. A. Bin Dehaish, X.-L. Qin, Weak convergence of a splitting algorithm in Hilbert spaces, J. Appl. Anal. Comput., 7 (2017), 427-438. 1

[10] S. Y. Cho, X.-L. Qin, L. Wang, Strong convergence of a splitting algorithm for treating monotone operators, Fixed Point Theory Appl., 2014 (2014), 15 pages. 1

[11] N.-N. Fang, Y.-P. Gong, Viscosity iterative methods for split variational inclusion problems and fixed point problems of a nonexpansive mapping, Commun. Optim. Theory, 2016 (2016), 15 pages. 1

[12] L. S. Liu, Ishikawa and Mann iterative process with errors for nonlinear strongly accretive mappings in Banach spaces, J. Math. Anal. Appl., 194 (1995), 114-125. 2.2

[13] A. Moudafi, E. Al-Shemas, Simultaneous iterative methods for split equality problem, Trans. Math. Program. Appl., 1 (2013), 1-11. 1, 3

[14] X.-L. Qin, S. Y. Cho, Convergence analysis of a monotone projection algorithm in reflexive Banach spaces, Acta Math. Sci. Ser. B Engl. Ed., 37 (2017), 488-502. 1

[15] X.-L. Qin, S. Y. Cho, L. Wang, A regularization method for treating zero points of the sum of two monotone operators, Fixed Point Theory Appl., 2014 (2014), 10 pages. 1

[16] X.-L. Qin, J.-C. Yao, Weak convergence of a Mann-like algorithm for nonexpansive and accretive operators, J. Inequal. Appl., 2016 (2016), 9 pages. 1

[17] D. R. Sahu, J. C. Yao, A generalized hybrid steepest descent method and applications, J. Nonlinear Var. Anal., 1 (2017), 111-126. 1

[18] J.-F. Tang, S.-S. Chang, J. Dong, Split equality fixed point problem for two quasi-asymptotically pseudocontractive mappings, J. Nonlinear Funct. Anal., 2017 (2017), 15 pages. 1

[19] H.-K. Xu, Inequalities in Banach spaces with applications, Nonlinear Anal., 16 (1991), 1127-1138. 2.1

[20] H.-Y. Zhou, P.-Y. Wang, Adaptively relaxed algorithms for solving the split feasibility problem with a new step size, J. Inequal. Appl., 2014 (2014), 22 pages. 1, 1 